\title{
A Lower Bound for the Rectilinear Crossing Number
}

Article in Graphs and Combinatorics - September 2005

DOI: $10.1007 / \mathrm{s} 00373-005-0612-5 \cdot$ Source: DBLP

CITATIONS

38

2 authors:

\section{Bernardo M. Ábrego}

California State University, Northridge

52 PUBLICATIONS 236 CITATIONS

SEE PROFILE
READS

41

Silvia Fernández-Merchant

California State University, Northridge

50 PUBLICATIONS 233 CITATIONS

SEE PROFILE

All content following this page was uploaded by Bernardo M. Ábrego on 11 September 2017. 


\title{
A Lower Bound for the Rectilinear Crossing Number.
}

\author{
Bernardo M. Ábrego, Silvia Fernández-Merchant \\ California State University Northridge \\ \{bernardo.abrego,silvia.fernandez\}@csun.edu \\ ver 4
}

\begin{abstract}
We give a new lower bound for the rectilinear crossing number $\overline{c r}(n)$ of the complete geometric graph $K_{n}$. We prove that $\overline{c r}(n) \geq \frac{1}{4}\left\lfloor\frac{n}{2}\right\rfloor\left\lfloor\frac{n-1}{2}\right\rfloor\left\lfloor\frac{n-2}{2}\right\rfloor\left\lfloor\frac{n-3}{2}\right\rfloor$ and we extend the proof of the result to pseudolinear drawings of $K_{n}$.
\end{abstract}

\section{Introduction}

The crossing number $\operatorname{cr}(G)$ of a simple graph $G$ is the minimum number of edge crossings in any drawing of $G$ in the plane, where each edge is a simple curve. The rectilinear crossing number $\overline{c r}(G)$ is the minimum number of edge crossings when $G$ is drawn in the plane using straight segments as edges. The crossing numbers have many applications to Discrete Geometry and Computer Science, see for example [7] and [9].

In this paper we study the problem of determining $\overline{c r}\left(K_{n}\right)$, where $K_{n}$ denotes the complete graph on $n$ vertices. For simplicity we write $\overline{c r}(n)=\overline{c r}\left(K_{n}\right)$. An equivalent formulation of the problem is to find the minimum number of convex quadrilaterals determined by $n$ points in general position (no three points on a line).

We mention here that $\operatorname{cr}\left(K_{n}\right)=\frac{1}{4}\left\lfloor\frac{n}{2}\right\rfloor\left\lfloor\frac{n-1}{2}\right\rfloor\left\lfloor\frac{n-2}{2}\right\rfloor\left\lfloor\frac{n-3}{2}\right\rfloor$ was conjectured by Zarankiewicz [12] and Guy [3], and there are (non-rectilinear) drawings of $K_{n}$ achieving this number. Of course $\operatorname{cr}\left(K_{n}\right) \leq \overline{c r}\left(K_{n}\right)$ but from the exact values of $\overline{c r}(n)$ for $n \leq 12$ [1], it is known that $\operatorname{cr}\left(K_{8}\right)<$ $\overline{c r}\left(K_{8}\right)$.

Jensen [6] and Singer [10] were the first to settle $\overline{c r}(n)=\Theta\left(n^{4}\right)$. In fact, since $\overline{c r}(5)=1$ then by an averaging argument it is easy to deduce that $\overline{c r}(n) \geq \frac{1}{5}\left(\begin{array}{c}n \\ 4\end{array}\right)$. This same idea was used by Brodsky et al [2] when they obtained $\overline{c r}(10)=62$, to deduce $\overline{c r}(n) \geq 0.3001\left(\begin{array}{l}n \\ 4\end{array}\right)$. Later Aicholzer et al [1] calculated $\overline{c r}(12)=153$ and used this to get $\overline{c r}(n) \geq 0.3115\left(\begin{array}{l}n \\ 4\end{array}\right)$. Very recently Wagner [11], following different methods proved $\overline{c r}(n) \geq 0.3288\left(\begin{array}{c}n \\ 4\end{array}\right)$. On the other hand Brodsky et al [2] constructed rectilinear drawings of $K_{n}$ showing $\overline{c r}(n) \leq \frac{6467}{16848}\left(\begin{array}{l}n \\ 4\end{array}\right) \leq 0.3838\left(\begin{array}{l}n \\ 4\end{array}\right)$. In this paper we prove the following theorem which gives as a lower bound for $\overline{c r}(n)$ the exact value conjectured by Zarankiewicz and Guy for $\operatorname{cr}\left(K_{n}\right)$.

Theorem $1 \overline{c r}(n) \geq \frac{1}{4}\left\lfloor\frac{n}{2}\right\rfloor\left\lfloor\frac{n-1}{2}\right\rfloor\left\lfloor\frac{n-2}{2}\right\rfloor\left\lfloor\frac{n-3}{2}\right\rfloor$.

It is known that $c^{*}=\lim _{n \rightarrow \infty} \overline{c r}(n) /\left(\begin{array}{l}n \\ 4\end{array}\right)>0$ exists. Our theorem gives $c^{*} \geq 3 / 8=0.375$ and it can in fact be generalized to a larger class of drawings of $K_{n}$. Namely, those obtained from the concept of simple allowable sequences of permutations introduced by Goodman and Pollack [4]. We denote by $\mathbb{P}^{2}$ the real projective plane, a pseudoline $\ell$ is a simple closed curve whose removal does not disconnect $\mathbb{P}^{2}$. A finite set $P$ in the plane is a generalized configuration if it consists of a set of points, together with a set of pseudolines joining each pair of points subject to the condition that each pseudoline intersects every other exactly once. If there is a single pseudoline for every pair then the generalized configuration is called simple. 
Consider a drawing of $K_{n}$ in the (projective) plane where each edge is represented by a simple curve. If each of these edges can be extended to a pseudoline in such a way that the resulting structure is a simple generalized configuration then we call such a drawing a pseudolinear drawing of $K_{n}$. We call pseudosegments the edges of a pseudolinear drawing. Clearly, every rectilinear drawing of $K_{n}$ is also pseudolinear. Thus the number $\widetilde{c r}(n)$, defined as the minimum number of edge crossings over all pseudolinear drawings of $K_{n}$, generalizes the quantity $\overline{c r}(n)$ and satisfies $\widetilde{c r}(n) \leq \overline{c r}(n)$. In this context we prove the following stronger result.

Theorem $2 \widetilde{c r}(n) \geq \frac{1}{4}\left\lfloor\frac{n}{2}\right\rfloor\left\lfloor\frac{n-1}{2}\right\rfloor\left\lfloor\frac{n-2}{2}\right\rfloor\left\lfloor\frac{n-3}{2}\right\rfloor$.

If a pseudolinear drawing is combinatorially equivalent to a rectilinear drawing then it is called stretchable. It is known that almost all pseudolinear drawings are non-stretchable. So it is conceivable that $\widetilde{c r}(n)<\overline{c r}(n)$ for $n$ sufficiently large, but at the moment we have no other evidence to support this. We also mention that the problem of determining whether a pseudolinear drawing is stretchable is NP-hard [8].

\section{Allowable Sequences}

Given a set $P$ of $n$ points in the plane, no three of them collinear, we construct the $\left(\left(\begin{array}{l}n \\ 2\end{array}\right)+1\right) \times n$ matrix $S(P)$ as follows.

Consider any circle $C$ containing $P$ in its interior. Let $\ell$ be the vertical right-hand side tangent line to $C$. We can assume without loss of generality that no segment in $P$ is perpendicular to $\ell$, we can also assume that no two segments in $P$ are parallel, otherwise we can perturb the set $P$ without changing the structure of its crossings. Label the points of $P$ from 1 to $n$ according to the order of their projections to $\ell, 1$ being the lowest and $n$ the highest. For each segment $\overline{i j}$ in $P$, let $c_{i j}=c_{j i}$ be the point in the upper half of $C$ such that the tangent line to $C$ at $c_{i j}$ is perpendicular to $i j$. This gives a linear order on the segments of $P$, inherited from the counter-clockwise order of the points $c_{i j}$ in $C$. Denote by $t_{r}$ the $r^{t h}$ pair of points (segment) in $P$ under this order. Indistinctly we use $t_{r}$ to denote an unordered pair $\{i, j\}$ or the point $c_{i j}=c_{j i}$. Using this, we recursively construct the matrix $S(P)$. The first row is $(1,2, \ldots, n)$, and the $(k+1)^{t h}$ row is obtained from the $k^{t h}$ row by switching the pair $t_{k} . S(P)$ is half a period of what is commonly referred as a circular sequence of permutations of $P$ [4].

$S(P)$ satisfies the following properties.

1. The first row of $S(P)$ is the $n$-tuple $(1,2,3, \ldots, n)$, the last row of $S(P)$ is the $n$-tuple $(n, n-1, \ldots, 2,1)$, and any row of $S(P)$ is a permutation of its first row.

2. Any row $r \geq 2$ is obtained from the previous row by switching two consecutive entries of the row $r-1$.

3. If the $r^{\text {th }}$ row is obtained by switching the entries $S_{r-1, c}$ and $S_{r-1, c+1}$ in the $(r-1)^{t h}$ row then $S_{r-1, c}<S_{r-1, c+1}$.

4. For every $1 \leq i<j \leq n$ there exists a unique row $1 \leq r \leq\left(\begin{array}{l}n \\ 2\end{array}\right)$ such that the entries $i$ and $j$ are switched from row $r$ to row $r+1$, i.e., $t_{r}=\{i, j\}, S_{r, c}=i<j<S_{r, c+1}$, and $S_{r+1, c}=j>i=S_{r+1, c+1}$ for some $1 \leq c \leq n-1$.

A simple allowable sequence of permutations is a combinatorial abstraction of a circular sequence of permutations associated with a configuration of points. It is defined as a doubly infinite periodic sequence of permutations of $1,2, \ldots, n$ satisfying that every permutation is obtained from the previous one by switching two adjacent numbers, and after $i$ and $j$ have been switched they do not switch again until all other pairs have switched. For the purposes of this paper we only use half a period of an allowable sequence. This translates to any $\left(\left(\begin{array}{l}n \\ 2\end{array}\right)+1\right) \times n$ matrix $S(P)$ satisfying properties 
1-4. From now on $S(P)$ will be such a matrix, not necessarily obtained as the circular sequence of permutations of a point set $P$.

It was proved by Goodman and Pollack [5] that every simple allowable sequence of permutations can be realized by a generalized configuration of points where the matrix $S(P)$ is determined by the cyclic order in which the connecting pseudolines meet a distinguished pseudoline (for example the pseudoline at infinity).

Next we establish when two pseudosegments do not intersect by means of the matrix $S(P)$. Given a simple generalized configuration of points $P$, we say that two pseudosegments $\widetilde{a b}$ and $\widetilde{c d}$ are separated if there exists a pseudoline in $P$ that leaves $\widetilde{a b}$ and $\widetilde{c d}$ in different sides. Note that any two non-incident pseudosegments (i.e., they do not share endpoints), either intersect in their interior (generate a crossing) or are separated. Thus $\widetilde{c r}\left(G_{P}\right)=\widetilde{c r}(P)$ is the number of non-incident pairs of pseudosegments minus the number of separated pseudosegments, where $G_{P}$ is a pseudolinear drawing of $K_{n}$ associated to $S(P)$.

Let $<_{r}$ be the linear order on $\{1,2,3, \ldots, n\}$ induced by the $r^{t h}$ row of $S(P)$. Observe that $\widetilde{a b}$ and $\tilde{c d}$ are separated if and only if there is a row $r$ such that $a, b<_{r} c, d$ or $c, d<_{r} a, b$. In this case we say $\widetilde{a b}$ and $\widetilde{c d}$ are separated in row $r$.

Lemma 3 allows us to count the number of separated pseudosegments in $P$. We say $\widetilde{a b}$ and $\tilde{c d}$ are neighbors in row $r$ if they are separated in row $r$ but not in row $r-1$.

Lemma $3 \widetilde{a b}$ and $\widetilde{c d}$ are separated if and only if there is a unique row $r$ where $\widetilde{a b}$ and $\tilde{c d}$ are neighbors.

Proof. First note that if $\tilde{a b}$ and $\tilde{c d}$ are neighbors, then they are separated by definition. Now assume $\widetilde{a b}$ and $\widetilde{c d}$ are separated, and let $R$ be the last row where they are separated. If $\widetilde{a b}$ and $\widetilde{c d}$ are separated in all rows above $R$ then they are separated in the first and consequently in the last rows, that is $R=\left(\begin{array}{l}n \\ 2\end{array}\right)+1$. This is impossible since having $\widetilde{a b}$ and $\widetilde{c d}$ separated in every row implies that they never reversed their order.

Consider the largest row $r \leq R$ such that $\widetilde{a b}$ and $\widetilde{c d}$ are not separated in row $r-1$. Then $\widetilde{a b}$ and $\widetilde{c d}$ are neighbors in row $r$. Finally, to prove that such a row is unique, let $r_{0}<r_{1}$ be two rows where $\widetilde{a b}$ and $c d$ are neighbors. Assume without loss of generality that $a<_{r_{0}} b<_{r_{0}} c<_{r_{0}} d$. Then $a<_{r_{0}-1} c<_{r_{0}-1} b<_{r_{0}-1} d$ and, since $b$ and $c$ switch exactly once, $b<_{r_{1}} c$. Also, by definition, one of the pairs $\widetilde{a c}, \widetilde{a d}$, or $\widetilde{b d}$ switches from row $r_{1}-1$ to row $r_{1}$. Since such a pair switches exactly once, then it has opposite orders in rows $r_{0}$ and $r_{1}$. Therefore one of the following should be satisfied

$$
b<_{r_{1}} c<_{r_{1}} a<_{r_{1}} d \text {, or } b<_{r_{1}} d<_{r_{1}} a<_{r_{1}} c \text {, or } a<_{r_{1}} d<_{r_{1}} b<_{r_{1}} c,
$$

but then $\widetilde{a b}$ and $\widetilde{c d}$ are not separated in row $r_{1}$.

For all $i \neq j$ in $P$, write $f_{P}(\widetilde{i j})=(r, c)$, if $i$ and $j$ switch in row $r$ and column $c$, that is $S_{r, c}=i=S_{r+1, c+1}$ and $S_{r, c+1}=j=S_{r+1, c}$. Note that this is well defined since the relative order of each pair of points $\{i, j\}$ in $P$ is changed exactly once.

For $1 \leq c \leq n-1$ define

$$
C_{P}(c)=\left\{r: \text { there exist } i, j \text { such that } f_{P}(\widetilde{i j})=(r, c)\right\},
$$

and let $\operatorname{ch}_{P}(c)=\operatorname{ch}(c)=\left|C_{P}(c)\right|$. In other words denotes the number of changes (switches) in column $c$.

Lemma 4 For any simple generalized configuration $P$ of $n$ points in the plane

$$
\widetilde{c r}(P)=3\left(\begin{array}{l}
n \\
4
\end{array}\right)-\sum_{j=1}^{n-1}(j-1)(n-1-j) \operatorname{ch}(j) .
$$


Proof. Since each four points in $P$ determine three pairs of non-incident pseudosegments, there are $3\left(\begin{array}{l}n \\ 4\end{array}\right)$ pairs of non-incident pseudosegments in $P$. It remains to prove that $\sum_{j=1}^{n-1}(j-1)(n-1-j) \operatorname{ch}(j)$ of these pairs are separated (non-crossing). Note that $\tilde{a b}$ and $\tilde{c d}$ are neighbors in row $r$ if and only if there are $x \in\{a, b\}, y \in\{c, d\}$ such that $x$ and $y$ switch from row $r-1$ to row $r$. By Lemma 3 , if $t_{r}=\{i, j\}$ and $i<j$ then all pairs $\widetilde{h j}$ and $\widetilde{i k}$ are neighbors (in row $r$ ) whenever $h<_{r} j$ and $i<_{r} k$. If $f_{P}(\widetilde{i j})=(r, c)$ then row $r$ accounts for $(c-1)(n-1-c)$ neighboring pairs of pseudosegments. Moreover, Lemma 3 guarantees that, when adding these quantities over all rows, we are counting all separated pairs of pseudosegments exactly once.

\section{Proof of Theorem 2}

Note that for fixed $1 \leq i \leq\left(\begin{array}{c}n \\ 2\end{array}\right), i$ switches exactly once with each number $j \neq i$, that is

$$
\left|\left\{f_{P}(\tilde{i j}): 1 \leq j \leq n, j \neq i\right\}\right|=n-1 .
$$

Moreover, since $n$ is the last entry in row 1 and the first entry in row $\left(\begin{array}{l}n \\ 2\end{array}\right)+1$, then when $i=n$ these $n-1$ switches occur in different columns, that is

$$
\left\{1 \leq c \leq n-1: f_{P}(\widetilde{n j})=(r, c) \text { for some } 1 \leq r \leq\left(\begin{array}{l}
n \\
2
\end{array}\right) \text {, and } 1 \leq j<n\right\}=\{1,2, \ldots, n-1\} .
$$

Therefore we can define $R_{P}(c)=r$ to be the unique row $r$ where the change of $n$ in column $c$ occurs, i.e., there exists $1 \leq j<n$ such that $f_{P}(\widetilde{n j})=(r, c)$. Also for $1 \leq c \leq n-1$ define the number of changes in column $c$ above and below row $R_{P}(c)$ as

$$
\begin{aligned}
& A_{P}(c)=\left\{r<R_{P}(c): \text { there exist } i, j \text { such that } f_{P}(\widetilde{i j})=(r, c)\right\} \\
& B_{P}(c)=\left\{r>R_{P}(c): \text { there exist } i, j \text { such that } f_{P}(\widetilde{i j})=(r, c)\right\} .
\end{aligned}
$$

The proof of the Theorem is based on the identity from Lemma 4, together with the next two lemmas. Let $m=\lfloor n / 2\rfloor$

Lemma 5 For any simple generalized configuration $P$ of $n$ points in the plane and $1 \leq k \leq m-1$ we have

$$
\left|A_{P}(k)\right|+\left|B_{P}(n-k)\right| \geq k .
$$

Proof. For $1 \leq j \leq k$ let

$$
\begin{aligned}
& g(j)=\min \left\{r: \text { there exists } i \text { such that } f_{P}(\widetilde{i j})=(r, k)\right\} \\
& h(j)=\min \left\{r: \text { there exists } i \text { such that } f_{P}(\widetilde{i j})=(r, n-k)\right\} .
\end{aligned}
$$

Since all $g(1), g(2), \ldots, g(k), h(1), h(2), \ldots, h(k)$ are different, and $A_{P}(k)$ and $B_{P}(n-k)$ are disjoint, then it is enough to prove that for all $1 \leq j \leq k$, either $h(j) \in B_{P}(n-k)$ or $g(j) \in A_{P}(k)$.

Assume that $h(j) \notin B_{P}(n-k)$. Then, since $h(j) \neq R_{P}(n-k), h(j)<R_{P}(n-k)$. Observe that $g(j)<h(j)$ and $R_{P}(n-k)<R_{P}(k)$ then

$$
g(j)<h(j)<R_{P}(n-k)<R_{P}(k) .
$$

Therefore $g(j) \in A_{P}(k)$. 
Lemma 6 For any simple generalized configuration $P$ of $n$ points in the plane and $1 \leq k \leq m-1$ we have

$$
\sum_{c=1}^{k}\left(\operatorname{ch}_{P}(c)+\operatorname{ch}_{P}(n-c)\right) \geq 3(1+2+3+\ldots+k)=3\left(\begin{array}{c}
k+1 \\
2
\end{array}\right) .
$$

Proof. By induction on $|P|=n$. The statement is true for $|P|=3$ by vacuity.

Consider the matrix $S(P)$ and let $P^{\prime}=P-\{n\}$. Note that $S\left(P^{\prime}\right)$ is the matrix obtained from erasing the unique entry equal to $n$ in each row of $S(P)$ and shifting one column left the necessary elements of $S(P)$. Also the rows where the corresponding change involves $n$ are deleted.

Note that for $1 \leq c \leq n-2$

$$
C_{P^{\prime}}(c)=A_{P}(c) \cup B_{P}(c+1)
$$

Thus for $1 \leq c \leq n-2$

$$
\operatorname{ch}_{P^{\prime}}(c)=\left|A_{P}(c)\right|+\left|B_{P}(c+1)\right| .
$$

Also notice that

$$
B_{P}(1)=A_{P}(n-1)=\varnothing
$$

and for $1 \leq c \leq n-1$

$$
\operatorname{ch}_{P}(c)=\left|A_{P}(c)\right|+\left|B_{P}(c)\right|+1 .
$$

Then by definition and (3)

$$
\begin{aligned}
\sum_{c=1}^{k}\left(\operatorname{ch}_{P}(c)+c h_{P}(n-c)\right) & =\sum_{c=1}^{k}\left(\left|A_{P}(c)\right|+\left|B_{P}(c)\right|+\left|A_{P}(n-c)\right|+\left|B_{P}(n-c)\right|+2\right) \\
& =2 k+\sum_{c=1}^{k}\left(\left|A_{P}(c)\right|+\left|B_{P}(c)\right|+\left|A_{P}(n-c)\right|+\left|B_{P}(n-c)\right|\right),
\end{aligned}
$$

separating one term from each sum we get

$$
\begin{aligned}
\sum_{c=1}^{k}\left(\operatorname{ch}_{P}(c)+\operatorname{ch}_{P}(n-c)\right)= & 2 k+\left|A_{P}(k)\right|+\left|B_{P}(1)\right|+\sum_{c=1}^{k-1}\left(\left|A_{P}(c)\right|+\left|B_{P}(c+1)\right|\right)+ \\
& +\left|A_{P}(n-1)\right|+\left|B_{P}(n-k)\right|+\sum_{c=2}^{k}\left(\left|A_{P}(n-c)\right|+\left|B_{P}(n-c+1)\right|\right),
\end{aligned}
$$

then by (1) and (2),

$$
\begin{aligned}
\sum_{c=1}^{k}\left(\operatorname{ch}_{P}(c)+\operatorname{ch}_{P}(n-c)\right) & =2 k+\left|A_{P}(k)\right|+\left|B_{P}(n-k)\right|+\sum_{c=1}^{k-1} \operatorname{ch}_{P^{\prime}}(c)+\sum_{c=2}^{k} \operatorname{ch}_{P^{\prime}}(n-c) \\
& =2 k+\left|A_{P}(k)\right|+\left|B_{P}(n-k)\right|+\sum_{c=1}^{k-1}\left(\operatorname{ch}_{P^{\prime}}(c)+\operatorname{ch}_{P^{\prime}}(n-1-c)\right) .
\end{aligned}
$$

Finally, by induction and Lemma 5 ,

$$
\begin{aligned}
\sum_{c=1}^{k}\left(c h_{P}(c)+\operatorname{ch}_{P}(n-c)\right) & \geq 2 k+k+3(1+2+\ldots+(k-1)) \\
& =3(1+2+\ldots+k)=3\left(\begin{array}{c}
k+1 \\
2
\end{array}\right) .
\end{aligned}
$$


Proof of Theorem 2. By Lemma 4, it is enough to find an upper bound for the expression

$$
\sum_{c=1}^{n-1}(c-1)(n-1-c) c h_{P}(c) \text {. }
$$

For $1 \leq j \leq m-1$ let $x_{j}=\operatorname{ch}_{P}(j)+c h_{P}(n-j)$, and $x_{m}=\operatorname{ch}_{P}(m)+\operatorname{ch}_{P}(m+1)$ if $n$ is odd, otherwise $x_{m}=c h_{P}(m)$. Under these definitions and according to Lemma 5 , together with the fact that $\sum_{j=1}^{m} x_{j}=\left(\begin{array}{c}n \\ 2\end{array}\right)$, it is enough to find the maximum of the function

$$
f\left(x_{1}, x_{2}, \ldots, x_{m}\right)=\sum_{j=1}^{m}(j-1)(n-1-j) x_{j}
$$

subject to the following linear conditions:

$$
\sum_{j=1}^{m} x_{j}=\left(\begin{array}{l}
n \\
2
\end{array}\right) \text { and } \sum_{j=1}^{k} x_{j} \geq 3\left(\begin{array}{c}
k+1 \\
2
\end{array}\right) \text { for every } 1 \leq k \leq m-1 .
$$

It is easy to see that the maximum occurs if and only if $x_{k}=3 k$ for all $1 \leq k \leq m-1$ and $x_{m}=\left(\begin{array}{c}n \\ 2\end{array}\right)-3\left(\begin{array}{c}m \\ 2\end{array}\right)$. If this is the case then

$$
f\left(x_{1}, x_{2}, \ldots, x_{m}\right)= \begin{cases}\frac{1}{64}(n-3)(n-1)\left(7 n^{2}-12 n-3\right) & \text { if } n \text { is odd } \\ \frac{1}{64} n(n-2)\left(7 n^{2}-26 n+16\right) & \text { if } n \text { is even. }\end{cases}
$$

Therefore, by Lemma 5, we conclude that

$$
\widetilde{c r}(P) \geq \begin{cases}\frac{1}{64}(n-3)^{2}(n-1)^{2} & \text { if } n \text { is odd } \\ \frac{1}{64} n(n-2)^{2}(n-4) & \text { if } n \text { is even. }\end{cases}
$$

i.e.,

$$
\widetilde{c r}(P) \geq \frac{1}{4}\left\lfloor\frac{n}{2}\right\rfloor\left\lfloor\frac{n-1}{2}\right\rfloor\left\lfloor\frac{n-2}{2}\right\rfloor\left\lfloor\frac{n-3}{2}\right\rfloor .
$$

\section{References}

[1] O. Aicholzer, F. Aurenhammer, and H. Krasser. On the crossing number of complete graphs. In Proc. 18th Ann ACM Symp Comp Geom., Barcelona Spain, 19-24, 2002.

[2] A. Brodsky, S. Durocher, and E. Gethner. Toward the Rectilinear Crossing Number of $K_{n}$ : New Drawings, Upper Bounds, and Asymptotics. Discrete Mathematics.

[3] R. K. Guy. The decline and fall of Zarankiewicz's theorem, in Proc. Proof Techniques in Graph Theory, (F. Harary ed.), Academic Press, N.Y., 63-69, 1969.

[4] J. E. Goodman and R. Pollack. Semispaces of configurations, cell complexes of arrangements in $P^{2}$. J. Combin. Theory Ser. A, 32: 1-19, 1982.

[5] J. E. Goodman and R. Pollack. A combinatorial version of the isotopy conjecture. In J. E. Goodman, E. Lutwak, J. Malkevitch, and R. Pollack, editors, Discrete Geometry and Convexity, pages 12-19, volume 440 of Ann. New York Acad. Sci., 1985. 
[6] H. F. Jensen. An upper bound for the rectilinear crossing number of the complete graph. $J$. Combin. Theory Ser B, 10: 212-216, 1971.

[7] J. Matoušek. Lectures on Discrete Geometry. Springer-Verlag, New York, N.Y., 2002.

[8] N.E. Mnëv. On manifolds of combinatorial types of projective configurations and convex polyhedra. Soviet Math. Dokl., 32: 335-337, 1985.

[9] J. Pach and G. Tóth. Thirteen problems on crossing numbers. Geombinatorics, 9: 194-207, 2000.

[10] D. Singer. Rectilinear crossing numbers. Manuscript, 1971.

[11] U. Wagner. On the Rectilinear Crossing Number of Complete Graphs.

[12] K. Zarankiewicz. On a problem of P. Turán concerning graphs, Fund. Math. 41: 137-145, 1954. 